\title{
Staphylococcus aureus resistente à meticilina nas Américas: revisão sistemática e metanálise da prevalência na pecuária
}

\author{
Silvio Barberato-Filho, ${ }^{1}$ Cristiane de Cássia Bergamaschi, ${ }^{1}$ Fernando de Sá Del Fiol, ${ }^{1}$ Felipe \\ Bernardini Antoniazzi, ${ }^{1}$ Julia Módolo Stievano, ${ }^{1}$ Ana Celine Justo, ${ }^{1}$ Camila de Paula Souza ${ }^{1}$ \\ e Marcus Tolentino Silva ${ }^{1}$
}

Como citar

Barberato-Filho S, Bergamaschi CC, Del Fiol FS, Antoniazzi FB, Stievano JM, Justo AC, et al. Staphylococcus aureus resistente à meticilina nas Américas: revisão sistemática e meta-análise da prevalência na pecuária. Rev Panam Salud Publica. 2020;44:e48. https://doi.org/10.26633/RPSP.2020.48

RESUMO

Objetivo. Determinar a prevalência de Staphylococcus aureus resistente à meticilina (MRSA) em animais destinados à produção de alimentos na região das Américas.

Métodos. Foi realizada uma revisão sistemática nas seguintes bases de dados: Scopus, Web of Science, MEDLINE, EMBASE e Biblioteca Virtual de Saúde (BVS). Foram selecionados artigos publicados nos últimos 10 anos, sem restrição quanto ao idioma. O desfecho de interesse foi a prevalência de MRSA em animais destinados à produção de alimentos. As prevalências foram agrupadas em metanálises de efeitos aleatórios pelo método de DerSimonian e Laird. A distribuição geográfica dos microrganismos resistentes e o decurso temporal das tendências de resistência também foram analisados.

Resultados. Dos 19 estudos incluídos, 11 foram conduzidos nos Estados Unidos e 11 analisaram amostras em suínos. Cinco estudos foram realizados na América do Sul. As amostras analisadas foram coletadas em locais de criação, de abate e de venda. A prevalência de MRSA na região das Américas foi de 7,6\% (IC95\%: 5,6 a 9,5\%) e apresentou-se maior em suínos [12,6\% (IC95\%: 7,0 a 18,2\%)], seguidos por bovinos [2,4\% (IC95\%: 1,2 a 3,7\%)] e aves [1,8\% (IC95\%: 0,3 a 3,4\%)]. Observou-se maior prevalência de MRSA na população de suínos da América do Norte e de bovinos da América Latina. Não houve variação significativa da prevalência de resistência nos 10 anos analisados.

Conclusões. A prevalência de MRSA em animais destinados à produção de alimentos na região das Américas foi maior em suínos, sem variação significativa da prevalência no decurso temporal.

Palavras-chave Resistência microbiana a medicamentos; Staphylococcus aureus resistente à meticilina; revisão sistemática; Américas.

O aumento no consumo de antimicrobianos para produção de proteína animal, tanto na profilaxia de doenças infecciosas como na promoção do crescimento, apresenta forte correlação com o aumento no número de microrganismos resistentes. Tal crescimento tornou-se um problema de saúde pública, com implicações para o tratamento e a prevenção de doenças infecciosas em humanos e animais $(1,2)$. Estima-se que a média de consumo anual de antimicrobianos por quilo de animal produzido chegue a $45 \mathrm{mg}$ para o gado de corte, $148 \mathrm{mg}$ para frangos e $172 \mathrm{mg}$ para suínos. Em 2010, o consumo mundial de antimicrobianos foi de 63 toneladas, com perspectiva de aumento para 105 em 2030 (3).

\footnotetext{
1 Universidade de Sorocaba, Programa de Pós-Graduação em Ciências Farmacêuticas, Sorocaba (SP), Brasil. $₫$ Marcus Tolentino Silva, marcusts@gmail.com
} 
O uso indiscriminado de antimicrobianos facilita a resistência microbiana, um mecanismo natural de adaptação dos microrganismos (4). Observa-se resistência a tetraciclinas, sulfonamidas e penicilinas frequentemente utilizadas em animais (5). Dentre os antimicrobianos críticos para a saúde humana, destaca-se a resistência a ciprofloxacino, eritromicina, cefalosporinas de terceira e quarta geração, linezolida e gentamicina (5). Além disso, a resistência pode resultar da troca de material genético entre organismos resistentes e não resistentes (6).

A meticilina é um antibiótico betalactâmico que, assim como a penicilina, se liga às proteínas que participam da síntese da parede celular, chamadas proteínas de ligação à penicilina (PLP), o que impede a formação da parede celular e resulta em lise bacteriana. Ao serem modificadas, essas proteínas conferem uma resistência à meticilina mediada pelo gene mecA positivo, que codifica a proteína de ligação à penicilina 2a (PLP 2a) (7). Cepas resistentes à meticilina da bactéria Staphylococcus aureus, caracterizada pela capacidade de rapidamente desenvolver resistência a qualquer tipo de antimicrobiano que venha a ser usado clinicamente ao longo de um tratamento (8), foram isoladas de bovinos, suínos, ovinos, aves, peixes e crustáceos nos locais de criação e de abate (9-11) e também em carnes cruas disponíveis no varejo $(12,13)$. Em poucos anos, o Staphylococcus aureus resistente à meticilina (MRSA) na pecuária se disseminou na produção de alimentos, com risco de infecção para os humanos. Os mais prejudicados são os criadores, trabalhadores de abatedouros e veterinários, que estão em frequente contato com animais portadores de MRSA (14).

Inexistem limites geográficos que impeçam a propagação da resistência microbiana mundial (15). Se as medidas preventivas e de contenção se restringirem a poucos países ou regiões, por exemplo, as políticas de contenção da resistência antimicrobiana não alcançarão seus objetivos (16). Nesse contexto, a Organização Mundial da Saúde (OMS) publicou um plano de ação global sobre resistência aos antimicrobianos (17), que também oferece uma lista de antimicrobianos criticamente importantes (18) e um guia de uso de antimicrobianos em animais de produção (19). O plano de ação global objetiva garantir, pelo maior tempo possível, a continuidade da capacidade de tratar e prevenir doenças infecciosas com medicamentos eficazes e seguros, com qualidade garantida, usados de maneira responsável e acessíveis a todos os que necessitam (17).

O objetivo da presente revisão sistemática foi determinar a prevalência de MRSA em animais destinados à produção de alimentos na região das Américas, bem como descrever a distribuição geográfica desses microrganismos resistentes e o decurso temporal das tendências de resistência.

\section{MATERIAIS E MÉTODOS}

Realizou-se uma revisão sistemática com metanálise de estudos de prevalência da resistência antimicrobiana em animais destinados à produção de alimentos. O protocolo da pesquisa está disponível no registro International Prospective Register of Systematic Reviews (PROSPERO) sob o número CRD42020160160.

Foram incluídos quaisquer estudos primários que relatassem dados de prevalência de MRSA em animais e estudos com foco em animais de produção infectados por cepas de MRSA na região das Américas. Os dados de prevalência foram obtidos a partir de amostras coletadas em locais de criação (fazendas, rebanhos, granjas, confinamentos, tanques e comunidades rurais), locais de abate (abatedouros), na indústria de processamento (laticínios e frigoríficos) e em pontos de venda (supermercados, açougues, varejo de alimentos, mercados atacadistas, feiras livres, peixarias e leilões).

Foram excluídos artigos apresentando dados oriundos de amostras obtidas de humanos (criadores, veterinários e outras pessoas em contato com o animal), dados obtidos em laboratório, estudos com informações incompletas sobre a prevalência de MRSA nos animais, estudos primários com abordagem qualitativa, resumos, livros e capítulos de livros, editoriais, cartas ao editor, inquéritos, revisões narrativas ou sistemáticas e panoramas descritivos.

Foram realizadas buscas nas seguintes bases de dados: SCOPUS (www.scopus.com), Web of Science (http://wokinfo. com/), MEDLINE (www.pubmed.gov), EMBASE (www. embase.com) e Biblioteca Virtual de Saúde (www.bvs.br), sem restrição quanto ao idioma, até outubro de 2018. Foram incluídos estudos publicados a partir de 2009 (últimos 10 anos). Tal restrição se justifica pela dinâmica da resistência, que pode variar significativamente ao longo do tempo.

Dois autores (JMS, CCB) revisaram a lista de referências de artigos de revisão, visando identificar outros estudos possivelmente elegíveis.

\section{Estratégia de busca}

A estratégia de busca foi elaborada pela combinação dos seguintes descritores (medical subject headings, MeSH) e seus sinônimos remissivos: para aves, poultry OR poultries OR (domestic Fowls) OR (domestic Fowl) OR Chickens OR Chicken OR (Gallus gallus domesticus) OR (Gallus domesticus) OR (Gallus gallus) OR Ducks OR Duck OR Geese OR goose OR gooses OR Turkey; para bovinos, cattle OR (Bos indicus) OR zebu OR zebus OR (Bos taurus) OR (Domestic Cow) OR (Domestic Cows) OR (Bos grunniens) OR Yak OR Yaks; para suínos, swine OR Suidae OR Pigs OR Warthogs OR (Wart Hogs) OR (Wart Hog) OR Phacochoerus OR (pig farming); para peixes, fishes OR Aquacultures OR Crustacea OR Crustaceas OR Ostracoda OR Ostracodas OR Ostracods OR Ostracod OR Aquiculture OR Aquicultures OR Seafood OR Seafoods OR (Sea-Food) OR (Sea Food) OR (Sea-Foods); e para ovinos, sheep OR Ovis OR (Dall Sheep) OR (Ovis dalli) OR (Domestic Sheep) OR (Ovis ammon aries) OR (Ovis aries) OR Mouflon OR Mouflons OR (Ovis gmelini musimon) OR (Ovis aries musimon) OR (sheep farming). Os termos relativos à resistência foram (Drug Resistance) OR (Microbial Drug Resistance) OR (Antimicrobial Drug Resistance) OR (Antimicrobial Drug Resistances) OR (Antibiotic Resistance). Finalmente, a estratégia de busca incluiu o termo methicillin-resistant Staphylococcus aureus.

\section{Determinação da elegibilidade}

Após exercício de calibração, quatro revisores (ACJ, FBA, JMS, CPS), em pares e de forma independente, avaliaram os títulos e resumos dos artigos identificados na busca de acordo com os critérios de elegibilidade. A mesma equipe de revisores aplicou esses critérios ao texto completo a fim de confirmar os estudos potencialmente elegíveis. As divergências foram resolvidas por um terceiro revisor (CCB, MTS ou SB-F). Utilizou-se a plataforma Covidence (https://www.covidence.org/home) para o gerenciamento dessa etapa, o que permitiu a remoção das duplicidades de registro. 


\section{Extração de dados}

Os revisores utilizaram um formulário padronizado e previamente testado de extração de dados, com instruções de registro. Quatro revisores (ACJ, FBA, JMS, CPS), de forma independente, fizeram um exercício de calibração pela extração de dados em pelo menos cinco artigos seguida de consenso. Esse procedimento foi repetido até que os revisores estivessem aptos a realizar a extração dos dados.

Foram extraídos os dados relativos ao continente e país onde o estudo foi realizado, ano do início da coleta, método laboratorial de deteç̧ão do MRSA (molecular, difusão em ágar e sistemas automatizados comerciais), material analisado (secreção nasal, fezes, carcaça, carne, leite, derivados do leite, equipamentos e demais fômites), ambiente (varejo e produção), número de amostras e número de cepas positivas de MRSA.

O risco de viés foi determinado com base no processo de seleção da amostra. Estudos sem sorteio da amostra foram considerados com alto risco de viés.

\section{Análise de dados}

O desfecho primário foi a prevalência de MRSA. Um sumário descritivo foi construído para apresentar os resultados encontrados e a variação entre os estudos. As análises dos dados foram realizadas pelo software estatístico STATA versão 14.2. As metanálises foram executadas pelo agrupamento de prevalências pelo modelo aleatório de DerSimonian e Laird e transformação de duplo arcoseno proposta por Freeman-Tukey para estabilizar as variâncias (20). A heterogeneidade foi investigada pelo cálculo do inverso da variância em modelo de efeito fixo, apresentada em porcentagem de $\mathrm{I}^{2}(21)$. A tendência temporal foi investigada por metarregressão das prevalências identificadas em duplo arcoseno no modelo de método de momentos, com a máxima verossimilhança restrita por modificação da variância dos coeficientes sugerida por Knapp e Hartung (22). Foram realizadas análises de subgrupo com informações por região (América do Norte e Latina), ambiente (produção e varejo), método laboratorial de detecção (difusão em ágar ou molecular ou ambos), presença de amostragem apropriada e antibiótico testado. Em todas as análises, foram considerados os intervalos de confiança de 95\% (IC95\%).

\section{RESULTADOS}

Foram incluídos 19 estudos com dados de prevalência de MRSA na região das Américas (23-41). Inicialmente, 2184 registros foram identificados nas bases de dados consultadas, dos quais 589 duplicatas foram removidas, somando 1595 resultados submetidos à triagem de título e resumo. Essa etapa resultou em 174 estudos submetidos à triagem de texto completo. Os motivos da exclusão estão descritos no fluxograma do processo de seleção (figura 1).

Dos 19 estudos selecionados, 17 (89,5\%) tiveram as amostras coletadas de 2009 a 2013, a maioria no ano de 2010. As amostras foram obtidas de aves $(n=6)$, bovinos $(n=8)$, suínos $(n=11)$ e ovinos $(n=1)$. Nenhum estudo avaliou a prevalência de MRSA em peixes ou crustáceos. Apenas cinco estudos (26,3\%) foram realizados na América do Sul, e um (5,3\%), na América Central. Os estudos incluíram amostras coletadas em locais de produção $(n=15)$ ou em pontos de venda no varejo $(n=14)$,
FIGURA 1. Fluxograma do processo de seleção da revisão sistemática

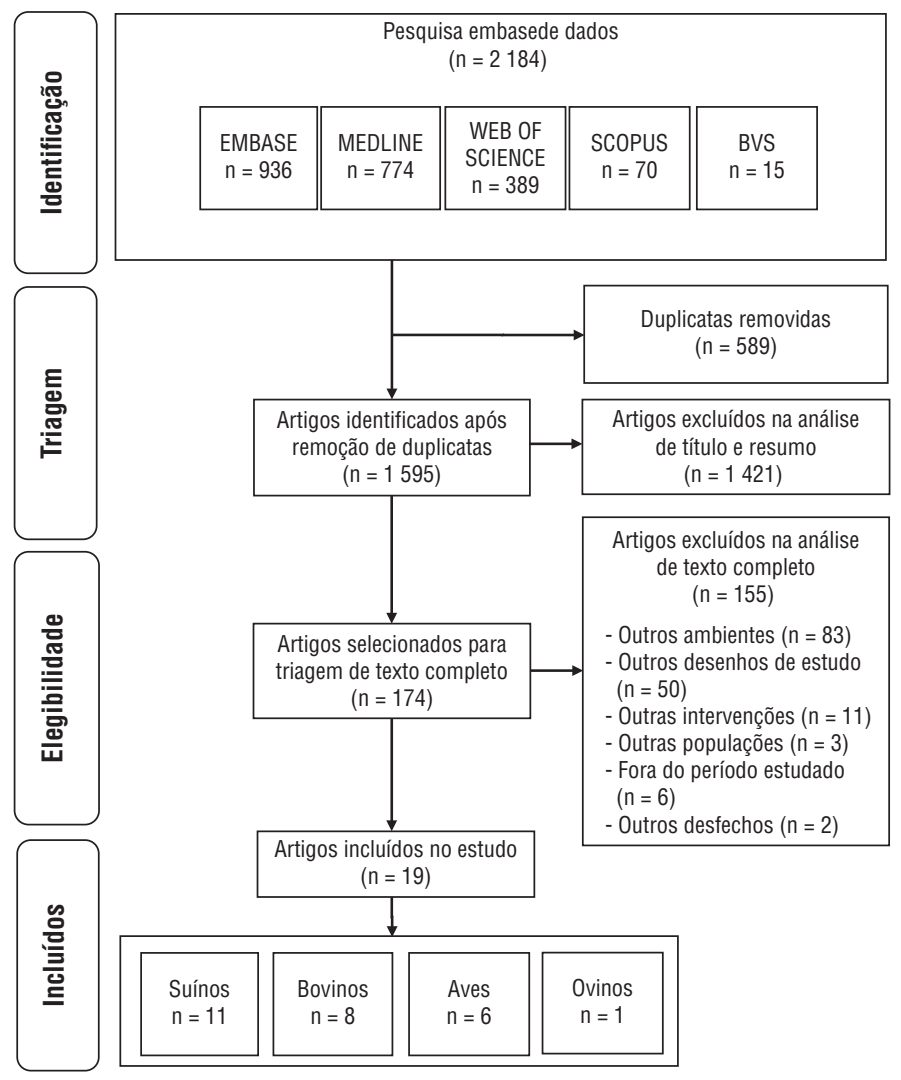

nos quais o principal material coletado foi carne $(\mathrm{n}=14)$. As características dos estudos incluídos estão descritas na tabela 1.

A prevalência de MRSA na região das Américas foi de 7,6\% (IC95\%: 5,6 a 9,5\%), sendo maior em suínos [12,6\% (IC95\%: 7,0 a $18,2 \%$ )], seguidos de bovinos [2,4\% (IC95\%: 1,2 a 3,7\%)] e aves [1,8\% (IC95\%: 0,3 a 3,4\%)] (figura 2). O único estudo realizado em ovinos (estado de Dakota do Norte, Estados Unidos) analisou 64 amostras de secreção nasal desses animais e não encontrou nenhuma cepa de MRSA.

As análises de subgrupos demonstraram maior prevalência de MRSA em suínos na América do Norte $(14,6 \%$ ) e em bovinos na América Latina (4,4\%). Também foi observada maior prevalência de MRSA em amostras coletadas nos locais de produção do que em pontos de venda (tabela 2).

Observou-se maior número de estudos com coleta de dados realizada no ano de 2010. A prevalência de MRSA foi ligeiramente superior nos primeiros anos do período investigado (figura 3).

\section{DISCUSSÃO}

Esta revisão sistemática determinou a prevalência de MRSA em animais destinados à produção de alimentos na região das Américas com base em 19 estudos, a maioria com amostras coletadas no ano de 2010 e nos Estados Unidos. As amostras foram obtidas principalmente em suínos, mas também em bovinos e aves; apenas um estudo foi realizado em ovinos. 
TABELA 1. Características dos estudos incluídos na revisão sistemática sobre Staphylococcus aureus resistente à meticilina nas Américas

\begin{tabular}{|c|c|c|c|c|c|}
\hline $\begin{array}{c}\text { Grupo e } \\
\text { referência }\end{array}$ & Coleta (ano) & País & Ambiente & $\begin{array}{c}\text { Material } \\
\text { analisado }\end{array}$ & $\begin{array}{c}\text { Risco de } \\
\text { viés }\end{array}$ \\
\hline \multicolumn{6}{|l|}{ Aves } \\
\hline 23 & 2010 & $\begin{array}{l}\text { Estados } \\
\text { Unidos }\end{array}$ & Varejo & Carne & Baixo \\
\hline 24 & 2009 & $\begin{array}{l}\text { Estados } \\
\text { Unidos }\end{array}$ & Varejo & Carne & Alto \\
\hline 25 & 2010 & $\begin{array}{l}\text { Estados } \\
\text { Unidos }\end{array}$ & Varejo & Carne & Alto \\
\hline 26 & 2010 & $\begin{array}{l}\text { Estados } \\
\text { Unidos }\end{array}$ & Varejo & Carne & Alto \\
\hline 27 & 2009 & $\begin{array}{l}\text { Estados } \\
\text { Unidos }\end{array}$ & Varejo & Carne & Alto \\
\hline 28 & 2014 & $\begin{array}{l}\text { Estados } \\
\text { Unidos }\end{array}$ & Varejo & Carne & Alto \\
\hline \multicolumn{6}{|l|}{ Bovinos } \\
\hline 25 & 2010 & $\begin{array}{l}\text { Estados } \\
\text { Unidos }\end{array}$ & $\begin{array}{l}\text { Produção/ } \\
\text { varejo }\end{array}$ & $\begin{array}{l}\text { - Secreção } \\
\text { nasal } \\
\text { - Carne }\end{array}$ & Alto \\
\hline 26 & 2010 & $\begin{array}{l}\text { Estados } \\
\text { Unidos }\end{array}$ & Varejo & Carne & Alto \\
\hline 29 & - & Brasil & Produção & Leite & Alto \\
\hline 27 & 2009 & $\begin{array}{l}\text { Estados } \\
\text { Unidos }\end{array}$ & Varejo & Carne & Alto \\
\hline 30 & 2009 & $\begin{array}{l}\text { Estados } \\
\text { Unidos }\end{array}$ & Produção & Leite & Baixo \\
\hline 31 & - & Brasil & Produção & Leite & Baixo \\
\hline 32 & 2012 & $\begin{array}{l}\text { Estados } \\
\text { Unidos }\end{array}$ & Varejo & Carne & Alto \\
\hline 33 & 2009 & Canadá & Produção & $\begin{array}{l}\text { - Secreção } \\
\text { nasal } \\
\text { - Fezes }\end{array}$ & Baixo \\
\hline \multicolumn{6}{|l|}{ Suínos } \\
\hline 34 & 2009 & Peru & Produção & $\begin{array}{l}\text { Secreção } \\
\text { nasal }\end{array}$ & Alto \\
\hline 35 & 2015 & Cuba & Produção & $\begin{array}{l}\text { Secreção } \\
\text { nasal }\end{array}$ & Alto \\
\hline 25 & 2010 & $\begin{array}{l}\text { Estados } \\
\text { Unidos }\end{array}$ & $\begin{array}{l}\text { Produção } \\
\text { Varejo }\end{array}$ & $\begin{array}{l}\text { - Secreção } \\
\text { nasal } \\
\text { - Carne }\end{array}$ & Alto \\
\hline 36 & 2010 & $\begin{array}{l}\text { Estados } \\
\text { Unidos }\end{array}$ & Produção & Secreção nasal & Alto \\
\hline 26 & 2010 & $\begin{array}{l}\text { Estados } \\
\text { Unidos }\end{array}$ & Varejo & Carne & Alto \\
\hline 27 & 2009 & $\begin{array}{l}\text { Estados } \\
\text { Unidos }\end{array}$ & Varejo & Carne & Alto \\
\hline 37 & 2010 & Uruguai & Produção & $\begin{array}{l}\text { Secreção } \\
\text { nasal }\end{array}$ & Alto \\
\hline 38 & 2009 & $\begin{array}{l}\text { Estados } \\
\text { Unidos }\end{array}$ & Produção & $\begin{array}{l}\text { - Secreção } \\
\text { nasal } \\
\text { - Fezes }\end{array}$ & Baixo \\
\hline 39 & 2010 & Canadá & $\begin{array}{l}\text { Produção/ } \\
\text { varejo }\end{array}$ & $\begin{array}{l}\text { - Carcaça } \\
\text { - Secreção } \\
\text { nasal } \\
\text { - Carne }\end{array}$ & Baixo \\
\hline 40 & 2009 & $\begin{array}{l}\text { Estados } \\
\text { Unidos }\end{array}$ & Produção & $\begin{array}{l}\text { Secreção } \\
\text { nasal }\end{array}$ & Baixo \\
\hline 41 & - & Brasil & Produção & Secreção nasal & Alto \\
\hline \multicolumn{6}{|l|}{ Ovinos } \\
\hline 25 & 2010 & $\begin{array}{l}\text { Estados } \\
\text { Unidos }\end{array}$ & Produção & $\begin{array}{l}\text { Secreção } \\
\text { nasal }\end{array}$ & Alto \\
\hline
\end{tabular}

A prevalência de MRSA foi maior em suínos, seguido de bovinos e aves. As análises de subgrupos demonstraram maior prevalência em suínos na América do Norte e em bovinos na América Latina. Uma metanálise anterior (42) sobre a prevalência de MRSA em aves e em carne de aves apresentou as seguintes taxas combinadas: 36\% (IC95\%: 1\% a 78\%) em perus; 13\% (IC95\%: 1\% a 28\%) em carne de peru; 5\% (IC95\%: $2 \%$ a 9\%) em frangos de corte; e 5\% (IC95\%: 3\% a 8\%) em carne de frango. A América do Sul teve a maior prevalência de MRSA (27\%; IC95\%: $17 \%$ a 37\%), e a América do Norte, a menor (1\%; IC95\%: $0 \%$ a 2\%). Nosso estudo aponta que, nos últimos 10 anos, a diminuição da prevalência de MRSA não foi estatisticamente significativa.

Porcos são o principal hospedeiro de MRSA, que tem sido causa crescente de infecções humanas em vários países. A Dinamarca, por exemplo, que monitora a prevalência de MRSA em suínos há mais de 10 anos, encontrou as seguintes taxas de prevalência nas fazendas de produção participantes nas pesquisas de 2008, 2010 e 2014, respectivamente: 3,5\% (7/198); 16,2\% (16/99); e 67,6\% (140/207). A proporção de fazendas de criação com teste positivo para MRSA aumentou de $0,0 \%(0 / 95)$ em 2008 para $71,8 \%(51 / 71)$ em 2014 (43).

Uma revisão sistemática com busca realizada em 2016 mapeou a prevalência global de MRSA em amostras de carne, com subgrupo por continente (44). Nas Américas, observou-se MRSA em 1,8\% (IC95\%: 0,2\% a 4,4\%) das amostras de bovinos, 0,4\% (IC95\%: 0,0\% a 2,0\%) de frangos e $4,2 \%$ (IC95\%: $1,9 \%$ a $7,3 \%)$ de suínos. Outra revisão sistemática do mesmo grupo encontrou prevalência de 1,6\% (IC95\%: 0,1\% a 4,5\%) em leite de vaca cru no continente americano (45). Dados de prevalência em leite de vaca pasteurizado ou fervido não foram encontrados.

Animais destinados à produção de alimentos (aves, bovinos, ovinos, peixes e suínos), além de serem considerados a principal fonte de MRSA, estão envolvidos na transferência de cepas de MRSA entre animais e humanos (e vice-versa) (46), com níveis elevados de resistência aos antimicrobianos utilizados na promoção do crescimento e na prevenção, controle e tratamento de doenças (47). A infecção por MRSA tornou-se um problema global de saúde pública e a presença de MRSA em animais tem como consequência o aumento do risco de doenças em humanos. A bacteremia por $S$. aureus exibe altas taxas de morbidade e mortalidade e pode causar infecções como pneumonia, osteomielite, endocardite, miocardite, pericardite e endocardite infecciosa ou sepse $(48,49)$.

Desde o ano de 2000, a produção de carne se estabilizou em países de alta renda, mas cresceu 68\%,64\% e 40\% na Ásia, África e América do Sul, respectivamente. Estima-se que 73\% do consumo global de todos os antimicrobianos esteja relacionado com a criação de animais destinados à produção de alimentos (5).

Em maio de 2015, a $68^{\text {a }}$ Assembleia Mundial da Saúde reconheceu a importância do problema, adotando o plano de ação global sobre resistência antimicrobiana. Impulsionadas por esse plano de ação global, foram publicadas, em 2017, as diretrizes da OMS recomendando a restrição do uso de antimicrobianos de importância médica na promoção do crescimento e na prevenção e tratamento de doenças infecciosas em animais destinados à produção de alimentos (19).

Conhecer a prevalência de MRSA em animais destinados à produção de alimentos na Região das Américas permite 


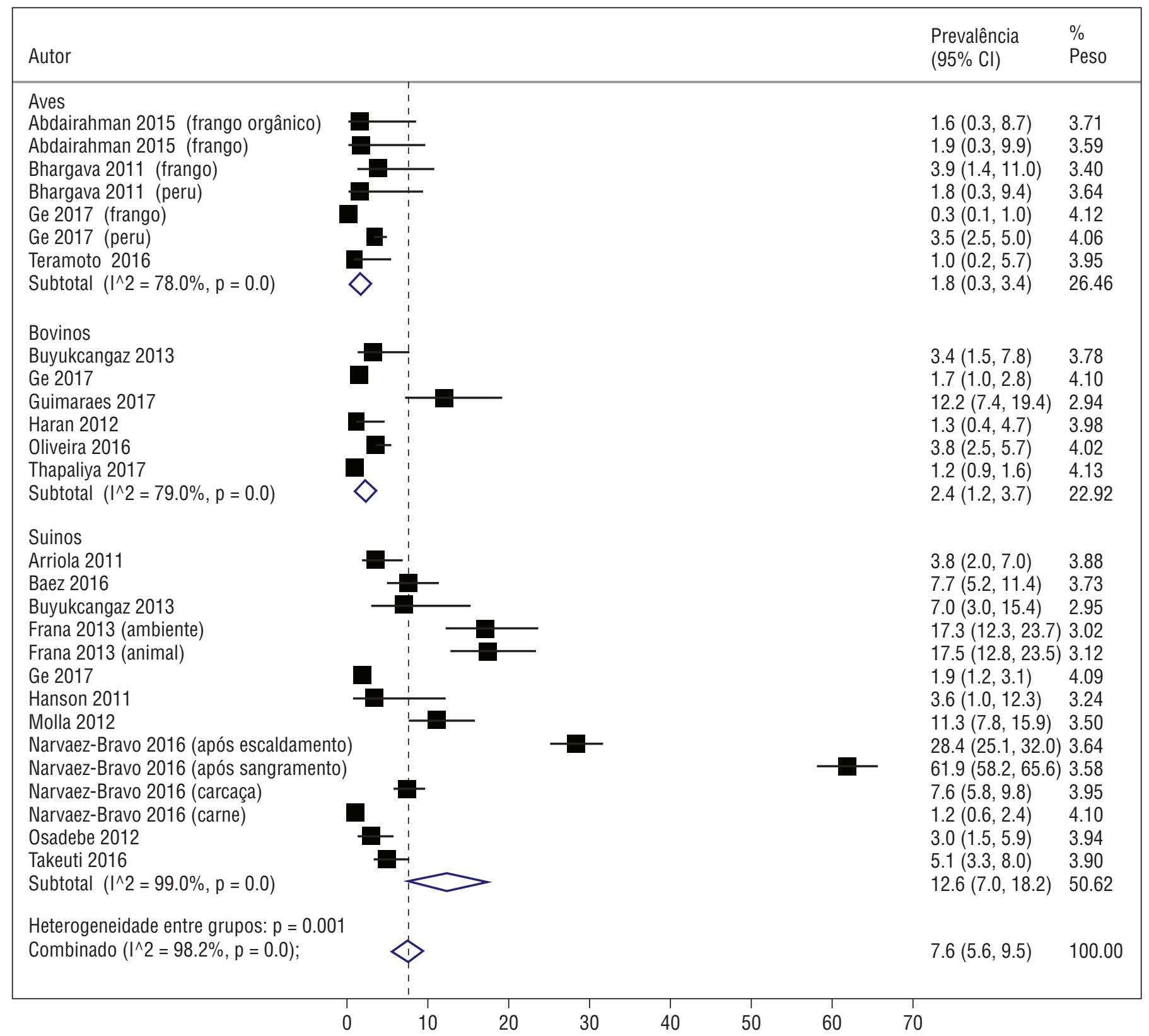

repensar o uso de antimicrobianos na criação de animais a fim de desenvolver alternativas e programas para redução do uso de antimicrobianos para o controle das enfermidades dos animais sem comprometer a cadeia produtiva, a partir do uso racional e prudente dessas substâncias e por meio de melhorias no manejo, na nutrição e nas boas práticas de produção.

$\mathrm{Na}$ presente revisão sistemática e metanálise, a heterogeneidade dos estudos e das amostras analisadas dificulta generalizações sobre a prevalência de MRSA na região das Américas. A comparação de resultados de amostras obtidas em fazendas de criação de animais em larga escala, com outras propriedades menores e amostras eventualmente restritas a animais de procriação ou animais em locais de abate pode influenciar os resultados. Nos locais de venda, a baixa prevalência de MRSA pode ser consequência de todas as amostras terem sido obtidas nos Estados Unidos e Canadá, sujeitos a rigorosos controles regulatórios e sanitários. Assim, existe possibilidade de os dados do presente estudo estarem subestimados. Apesar disso, a reunião de dados de prevalência de MRSA na região das Américas a partir de 19 estudos primários, realizados em seis países, nos últimos 10 anos, com resultados de prevalência em locais de produção e de venda, representa uma contribuição útil para a saúde pública e a realização de estudos futuros.

Os resultados deste estudo reforçam a necessidade de planejar intervenções que possam reduzir o uso de antimicrobianos na criação de animais destinados à produção de alimentos na Região das Américas e aumentar os cuidados no manejo dos 
TABELA 2. Metanálise da prevalência de Staphylococcus aureus resistente à meticilina em subgrupos selecionados

\begin{tabular}{|c|c|c|c|}
\hline \multirow[b]{2}{*}{ Subgrupo } & \multicolumn{3}{|c|}{$\%(I C 95 \%)^{a}$} \\
\hline & Aves & Bovinos & Suínos \\
\hline \multicolumn{4}{|l|}{ Região } \\
\hline América do Norte & $1,8(0,3$ a 3,4$)$ & $1,3(0,9$ a 1,7$)$ & $14,6(7,6$ a 21,5$)$ \\
\hline América Latina & & $4,4(2,8$ a 5,9$)$ & $5,3(3,3$ a 7,4$)$ \\
\hline \multicolumn{4}{|l|}{ Ambiente } \\
\hline Locais de produção & & $4,5(0,9$ a 8,1$)$ & $16,3$ (6,7 a 25,9$)$ \\
\hline Varejo & $1,8(0,3$ a 3,4$)$ & $1,5(0,8$ a 2,1$)$ & $1,8(0,8$ a 2,8$)$ \\
\hline \multicolumn{4}{|l|}{ Método laboratorial } \\
\hline Difusão em ágar & $1,7(0,3$ a 3,0$)$ & $1,2(0,9$ a 1,6$)$ & $5,6(2,0$ a 9,1$)$ \\
\hline Detecção molecular em PCR & & & $5,1(3,3$ a 8,0$)$ \\
\hline Difusão em ágar + molecular & $0,6(0,3$ a 1,0$)$ & $3,2(1,1$ a 5,3$)$ & $15,4(7,9$ a 22,8$)$ \\
\hline Amostragem apropriada & $1,7(0,0$ a 4,2$)$ & $2,7(1,5$ a 3,9$)$ & $18,8(5,5$ a 32,2$)$ \\
\hline \multicolumn{4}{|l|}{ Antibiótico testado } \\
\hline Meticilina & $1,3(0,0$ a 2,9$)$ & $1,3(0,7$ a 1,9$)$ & $5,1(3,3$ a 8,0$)$ \\
\hline Oxacilina & $0,6(0,3$ a 1,0$)$ & $4,3(1,2$ a 7,4$)$ & $11,7(2,9$ a 20,6$)$ \\
\hline
\end{tabular}

a \% (IC95\%): prevalência e intervalo de confiança de $95 \%$.

'PCR: reação em cadeia da polimerase.

animais, na manipulação e no processamento da carne e derivados, estabelecendo e fiscalizando padrões sanitários rigorosos, que restrinjam a disseminação de cepas resistentes, capazes de causar danos à saúde humana e dos animais.

Contribuição dos autores. SB-F, CCB, FDF e MTS elaboraram o projeto de pesquisa, analisaram e interpretaram os resultados e redigiram o manuscrito. FBA, JMS, ACJ e CPS selecionaram os estudos, realizaram a extração de dados e descreveram os resultados. Todos os autores revisaram o manuscrito e aprovaram a versão final.
FIGURA 3. Análise da influência do ano de coleta nas estimativas de prevalência de Staphylococcus aureus resistente à meticilina (metarregressão) nas Américas

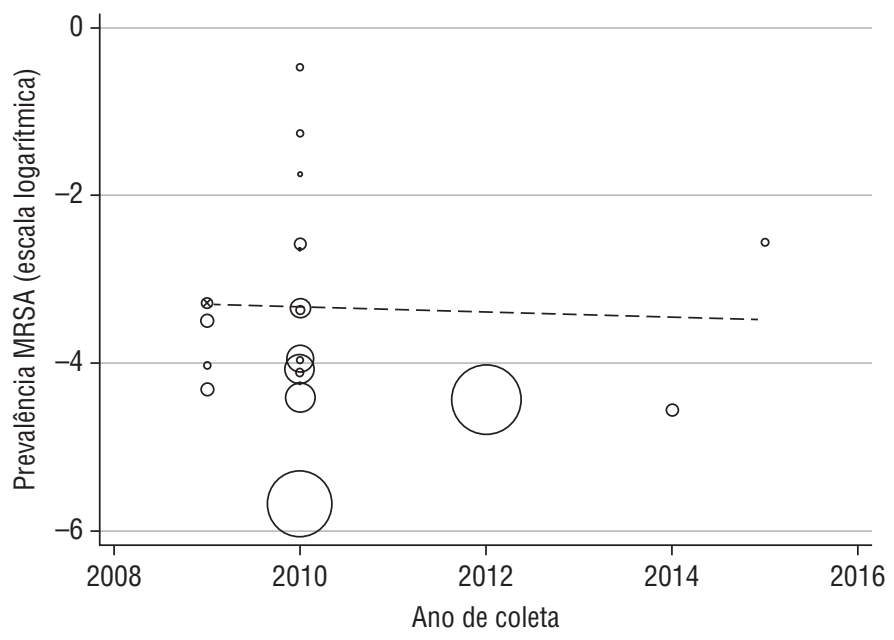

Conflitos de interesse. Nada declarado pelos autores.

Financiamento. FBA, JMS e ACJ receberam bolsa de iniciação científica da Universidade de Sorocaba. CPS recebeu bolsa de iniciação científica do Conselho Nacional de Desenvolvimento Científico e Tecnológico (CNPq).

Declaração. As opiniões expressas no manuscrito são de responsabilidade exclusiva dos autores e não refletem necessariamente a opinião ou política da RPSP/PAJPH ou da Organização Pan-Americana da Saúde (OPAS).

\section{REFERÊNCIAS}

1. Limayem A, Donofrio RS, Zhang C, Haller E, Johnson MG. Studies on the drug resistance profile of Enterococcus faecium distributed from poultry retailers to hospitals. J Environ Sci Health B. 2015;50(11):827-32.

2. Chantziaras I, Boyen F, Callens B, Dewulf J. Correlation between veterinary antimicrobial use and antimicrobial resistance in food-producing animals: a report on seven countries. J Antimicrob Chemother. 2014;69(3):827-34.

3. Van Boeckel TP, Brower C, Gilbert M, Grenfell BT, Levin SA, Robinson TP, et al. Global trends in antimicrobial use in food animals. Proc Natl Acad Sci U S A. 2015;112(18):5649-54.

4 . Beovic B. The issue of antimicrobial resistance in human medicine. Int J Food Microbiol. 2006;112(3):280-7.

5. Van Boeckel TP, Pires J, Silvester R, Zhao C, Song J, Criscuolo NG, et al. Global trends in antimicrobial resistance in animals in lowand middle-income countries. Science. 2019;365(6459):eaaw1944.

6. Perez-Rodriguez F, Mercanoglu Taban B. A State-of-Art Review on Multi-Drug Resistant Pathogens in Foods of Animal Origin: Risk Factors and Mitigation Strategies. Front Microbiol. 2019;10:2091.

7. Chambers HF. Methicillin resistance in staphylococci: molecular and biochemical basis and clinical implications. Clin Microbiol Rev. 1997;10(4):781-91.

8. Pantosti A. Methicillin-Resistant Staphylococcus aureus Associated with Animals and Its Relevance to Human Health. Front Microbiol. 2012;3:127.

9. de Neeling AJ, van den Broek MJ, Spalburg EC, van Santen-Verheuvel MG, Dam-Deisz WD, Boshuizen HC, et al. High prevalence of methicillin resistant Staphylococcus aureus in pigs. Vet Microbiol. 2007;122(3-4):366-72.
10. Rao RT, Jayakumar K, Kumar P. Bovine origin Staphylococcus aureus: A new zoonotic agent? Vet World. 2017;10(10):1275-80.

11. Soltan Dallal MM MNFR, Sharifi-Yazdi MK. . Prevalence of sea, seb, tsst and mecA genes in Staphylococcus aureus isolated from shrimps sold in seafood retailers in Tehran, Iran. Journal of Food Quality and Hazards Control. 2018; 5:72-76. 2018.

12. Velasco V, Vergara JL, Bonilla AM, Munoz J, Mallea A, Vallejos D, et al. Prevalence and Characterization of Staphylococcus aureus Strains in the Pork Chain Supply in Chile. Foodborne Pathog Dis. 2018;15(5):262-8.

13. Vaiyapuri M, Joseph TC, Rao BM, Lalitha KV, Prasad MM. Methicillin-Resistant Staphylococcus aureus in Seafood: Prevalence, Laboratory Detection, Clonal Nature, and Control in Seafood Chain. J Food Sci. 2019;84(12):3341-51.

14. Huijsdens XW, van Dijke BJ, Spalburg E, van Santen-Verheuvel MG, Heck ME, Pluister GN, et al. Community-acquired MRSA and pig-farming. Ann Clin Microbiol Antimicrob. 2006;5:26.

15. Acar JF, Moulin G. Integrating animal health surveillance and food safety: the issue of antimicrobial resistance. Rev Sci Tech. 2013;32(2):383-92.

16. Founou LL, Founou RC, Essack SY. Antibiotic Resistance in the Food Chain: A Developing Country-Perspective. Front Microbiol. 2016;7:1881.

17. World Health Organization. Global action plan on antimicrobial resistance. 2015. Disponível em: https://apps.who.int/iris/ handle/10665/193736.

18. WHO. Critically Important Antimicrobials for Human Medicine: 6th Revision 2018: Ranking of medically important antimicrobials 
for risk management of antimicrobial resistance due to non-human use.: World Health Organization; 2018.

19. WHO. WHO guidelines on use of medically important antimicrobials in food-producing animals. Geneva: World Health Organization; 2017. Disponível em: https://apps.who.int/iris/ bitstream/handle/10665/258970/9789241550130-eng.pdf;jsessioni$\mathrm{d}=6$ C321D4D00304C0F3D08247A30266934? sequence $=1$.

20. Barendregt JJ, Doi SA, Lee YY, Norman RE, Vos T. Meta-analysis of prevalence. J Epidemiol Community Health. 2013;67(11):974-8.

21. Nyaga VN, Arbyn M, Aerts M. Metaprop: a Stata command to perform meta-analysis of binomial data. Arch Public Health. 2014;72(1):39.

22. Knapp G, Hartung J. Improved tests for a random effects metaregression with a single covariate. Statistics in medicine. 2003;22(17):2693-710.

23. Abdalrahman LS, Stanley A, Wells H, Fakhr MK. Isolation, Virulence, and Antimicrobial Resistance of Methicillin-Resistant Staphylococcus aureus (MRSA) and Methicillin Sensitive Staphylococcus aureus (MSSA) Strains from Oklahoma Retail Poultry Meats. Int J Environ Res Public Health. 2015;12(6):6148-61.

24. Bhargava K, Wang X, Donabedian S, Zervos M, de Rocha L, Zhang Y. Methicillin-resistant Staphylococcus aureus in retail meat, Detroit, Michigan, USA. Emerg Infect Dis. 2011;17(6):1135-7.

25. Buyukcangaz E, Velasco V, Sherwood JS, Stepan RM, Koslofsky RJ, Logue CM. Molecular typing of Staphylococcus aureus and methicillin-resistant S. aureus (MRSA) isolated from animals and retail meat in North Dakota, United States. Foodborne Pathog Dis. 2013;10(7):608-17.

26. Ge BL, Mukherjee S, Hsu CH, Davis JA, Tran TTT, Yang Q, et al. MRSA and multidrug-resistant Staphylococcus aureus in US retail meats, 2010-2011. Food Microbiol. 2017;62:289-97.

27. Hanson BM, Dressler AE, Harper AL, Scheibel RP, Wardyn SE, Roberts LK, et al. Prevalence of Staphylococcus aureus and methicillin-resistant Staphylococcus aureus (MRSA) on retail meat in Iowa. Journal of Infection and Public Health. 2011;4(4):169-74.

28. Teramoto H, Salaheen S, Biswas D. Contamination of post-harvest poultry products with multidrug resistant Staphylococcus aureus in Maryland-Washington DC metro area. Food Control. 2016;65:132-5.

29. Guimaraes FF, Manzi MP, Joaquim SF, Richini-Pereira VB, Langoni $\mathrm{H}$. Short communication: Outbreak of methicillin-resistant Staphylococcus aureus (MRSA)-associated mastitis in a closed dairy herd. J Dairy Sci. 2017;100(1):726-30.

30. Haran KP, Godden SM, Boxrud D, Jawahir S, Bender JB, Sreevatsan S. Prevalence and characterization of Staphylococcus aureus, including methicillin-resistant Staphylococcus aureus, isolated from bulk tank milk from Minnesota dairy farms. J Clin Microbiol. 2012;50(3):688-95.

31. Oliveira CJB, Tiao N, de Sousa FGC, de Moura JFP, Santos Filho L, Gebreyes WA. Methicillin-Resistant Staphylococcus aureus from Brazilian Dairy Farms and Identification of Novel Sequence Types. Zoonoses and Public Health. 2016;63(2):97-105.

32. Thapaliya D, Forshey BM, Kadariya J, Quick MK, Farina S, A OB, et al. Prevalence and molecular characterization of Staphylococcus aureus in commercially available meat over a one-year period in Iowa, USA. Food Microbiol. 2017;65:122-9.

33. Weese JS, Hannon SJ, Booker CW, Gow S, Avery BP, Reid-Smith RJ. The prevalence of methicillin-resistant Staphylococcus aureus colonization in feedlot cattle. Zoonoses and public health. 2012;59(2):144-7.

34. Arriola CS, Güere ME, Larsen J, Skov RL, Gilman RH, Gonzalez AE, et al. Presence of methicillin-resistant staphylococcus aureus in pigs in Peru. PLoS One. 2011;6(12).
35. Baez M, Collaud A, Espinosa I, Perreten V. MRSA clone USA 300 in pigs from Cuba. Clin Chem Lab Med. 2016;54(7):eA61.

36. Frana TS, Beahm AR, Hanson BM, Kinyon JM, Layman LL, Karriker LA, et al. Isolation and Characterization of Methicillin-Resistant Staphylococcus aureus from Pork Farms and Visiting Veterinary Students. PLoS One. 2013;8(1):10.

37. Meyer C, Fredriksson-Ahomaa M, Stuber E, Thiel S, Martlbauer E. High Frequency of Multiresistant Coagulase-Positive Staphylococcus aureus Found in Slaughter Pigs in Uruguay. Foodborne Pathog Dis. 2012;9(1):86-90.

38. Molla B, Byrne M, Abley M, Mathews J, Jackson CR, Fedorka-Cray $\mathrm{P}$, et al. Epidemiology and genotypic characteristics of methicillinresistant Staphylococcus aureus strains of porcine origin. J Clin Microbiol. 2012;50(11):3687-93.

39. Narvaez-Bravo C, Toufeer M, Weese SJ, Diarra MS, Deckert AE, Reid-Smith R, et al. Prevalence of methicillin-resistant Staphylococcus aureus in Canadian commercial pork processing plants. J Appl Microbiol. 2016;120(3):770-80.

40. Osadebe LU, Hanson B, Smith TC, Heimer R. Prevalence and Characteristics of Staphylococcus aureus in Connecticut Swine and Swine Farmers. Zoonoses Public Health. 2013;60(3):234-43.

41. Takeuti KL, Malgarin CM, Amaral AF, de Barcellos D. Frequency of Methicillin-Resistant Staphylococcus aureus (MRSA) in Fattening Pigs in the State of Rio Grande do Sul, Brazil. Acta Sci Vet. 2016;44:4

42. Ribeiro CM, Stefani LM, Lucheis SB, Okano W, Cruz JCM, Souza GV, et al. Methicillin-Resistant Staphylococcus aureus in Poultry and Poultry Meat: A Meta-Analysis. J Food Prot. 2018;81(7):1055-62.

43. Sieber RN, Skov RL, Nielsen J, Schulz J, Price LB, Aarestrup FM, et al. Drivers and Dynamics of Methicillin-Resistant LivestockAssociated Staphylococcus aureus CC398 in Pigs and Humans in Denmark. MBio. 2018;9(6)

44. Ou Q, Peng Y, Lin D, Bai C, Zhang T, Lin J, et al. A Meta-Analysis of the Global Prevalence Rates of Staphylococcus aureus and Methicillin-Resistant S. aureus Contamination of Different Raw Meat Products. J Food Prot. 2017:763-74.

45. Ou Q, Zhou J, Lin D, Bai C, Zhang T, Lin J, et al. A large meta-analysis of the global prevalence rates of $S$. aureus and MRSA contamination of milk. Crit Rev Food Sci Nutr. 2018;58(13):2213-28.

46. Vanderhaeghen W, Cerpentier T, Adriaensen C, Vicca J, Hermans K, Butaye P. Methicillin-resistant Staphylococcus aureus (MRSA) ST398 associated with clinical and subclinical mastitis in Belgian cows. Vet Microbiol. 2010;144(1-2):166-71.

47. DeDonder KD, Apley MD. A literature review of antimicrobial resistance in Pathogens associated with bovine respiratory disease. Anim Health Res Rev. 2015;16(2):125-34.

48. Hassoun A, Linden PK, Friedman B. Incidence, prevalence, and management of MRSA bacteremia across patient populations-a review of recent developments in MRSA management and treatment. Crit Care. 2017;21(1):211.

49. Osman K, Alvarez-Ordonez A, Ruiz L, Badr J, ElHofy F, Al-Maary $\mathrm{KS}$, et al. Antimicrobial resistance and virulence characterization of Staphylococcus aureus and coagulase-negative staphylococci from imported beef meat. Ann Clin Microbiol Antimicrob. 2017;16(1):35.

Manuscrito recebido em 25 de outubro de 2019. Aceito em versão revisada em 11 de março de 2020 


\section{Methicillin-resistant Staphylococcus aureus in the Americas: systematic review and metanalysis of prevalence in food-producing animals}

ABSTRACT Objective. To determine the prevalence of methicillin-resistant Staphylococcus aureus (MRSA) in animals used to produce foods in the Americas.

Method. A systematic literature review was performed in the following databases: Scopus, Web of Science, MEDLINE, EMBASE, and Virtual Health Library. Articles published in the past 10 years, without language limits, were selected. The outcome of interest was the prevalence of MRSA in food-producing animals. Prevalence rates were meta-analyzed in grouped random effects models using the DerSimonian and Laird method. The geographic distribution of MRSA and the time trend of resistance were also analyzed.

Results. Of 19 studies included, 11 were performed in the United States and 11 analyzed pig samples. Five studies were performed in South America. The samples analyzed in the studies were collected in farming, processing, and retail sites. MRSA prevalence in the Americas was 7.6\% (95\%Cl: 5.6-9.5\%), and was higher in pigs [12.6\% $(95 \% \mathrm{Cl}: 7.0-18.2 \%)]$ followed by bovine cattle [2.4\% (95\%Cl: $1.2-3.7 \%)]$ and poultry [1.8\% (95Cl\%: 0.3-3.4\%)]. MRSA prevalence was higher in pigs in North America and bovine cattle in Latin America. There was no significant variation in MRSH prevalence along the 10-year period analyzed.

Conclusions. MRSA prevalence in food-producing animals in the Americas was higher in pigs, without significant changes across time.

Keywords Drug resistance, microbial; methicillin-resistant Staphylococcus aureus; Americas; systematic review.

Staphylococcus aureus resistente a la meticilina en la Región de las Américas: revisión sistemática y metanálisis de la prevalencia en la actividad agropecuaria

RESUMEN Objetivo. Determinar la prevalencia de Staphylococcus aureus resistente a la meticilina (MRSA por su sigla en inglés) en animales destinados a la producción de alimentos en la Región de las Américas.

Métodos. Se realizó una revisión sistemática en las siguientes bases de datos: Scopus, Web of Science, MEDLINE, EMBASE y Biblioteca Virtual de Salud (BVS). Se seleccionaron artículos publicados en los últimos diez años, sin restricción en cuanto al idioma. El resultado de interés fue la prevalencia de MRSA en animales destinados a la producción de alimentos. Las tasas de prevalencia se agruparon en metanálisis de efectos aleatorios por el método de DerSimonian y Laird. También se analizaron la distribución geográfica de los microorganismos resistentes y las tendencias de resistencia con el transcurso del tiempo.

Resultados. De los 19 estudios incluidos, 11 se realizaron en Estados Unidos y en 11 se analizaron muestras tomadas de cerdos. Se efectuaron cinco estudios en América del Sur. Las muestras analizadas se recogieron en lugares de cría, sacrificio y venta. La prevalencia de MRSA en la Región de las Américas fue de 7,6\% (IC95\%: 5,6-9,5\%) con una tasa mayor en cerdos [12,6\% (IC95\%: 7,0-18,2\%)], seguidos por bovinos [2,4\% (IC95\%: 1,2-3,7\%)] y aves [1,8\% (IC95\%: 0,3-3,4\%)]. Se observó una mayor prevalencia de MRSA en la población porcina de América del Norte y en la población bovina de América Latina. No hubo variación significativa de la prevalencia de resistencia en los diez años analizados.

Conclusiones. La prevalencia de MRSA en animales destinados a la producción de alimentos en la Región de las Américas fue mayor en cerdos, sin variación significativa de la prevalencia con el transcurso del tiempo.

Palabras clave Farmacorresistencia microbiana; Staphylococcus aureus resistente a meticilina; Américas; revisión sistemática. 